\title{
Semiconductor Cavity Quantum Electrodynamics with Single Quantum Dots
}

\author{
S. Reitzenstein, C. Schneider, S. Münch, C. Kistner, M. Strauss, A. Huggenberger, \\ P. Franeck, P. Weinmann, M. Kamp, S. Höfling*, L. Worschech and A. Forchel \\ Technische Physik, Physikalisches Institut, Wilhelm Conrad Röntgen Research Center for Complex Material \\ Systems, Universität Würzburg, Am Hubland, D-97074 Würzburg, Germany
}

This paper summarizes recent progress achieved in the field of semiconductor cavity quantum electrodynamics with single quantum dots with the focus being on micropillar cavities. Light-matter interaction both in the strong and weak coupling regime is presented. Resonance tuning of the quantum dot by temperature, electric fields and magnetic fields is demonstrated while the strong coupling regime can be reached. Additionally, deterministic device integration of single positioned quantum dots is reported by a combination of site controlled quantum dot growth via directed nucleation and subsequent device alignment to overcome the degree of randomness of the quantum dot position in so far most common quantum dot-cavity systems.

PACS numbers: 73.21.La, 71.35.Ji, 71.55.Eq, 37.30.+i

\section{Introduction}

Cavity quantum electrodynamics (CQED) based on a solid state platform is a highly prospering research field [1]. The exploitation of single semiconductor quantum dots (QDs) as zero-dimensional light emitters and artificial atoms and their integration into optical resonators has led to an entire new generation of highly sophisticated devices in semiconductor systems. For instance, efficient single photon sources [2-7] and few to single QD lasers [8-11] have been extensively researched. Furthermore, the manipulation of a single electron spin in a self-assembled QD [12] is an important first step toward cavity based future quantum information processing schemes based on QDs [13, 14]. Especially the rather straightforward way of electrical current injection into doped low-mode volume GaAs based structures can pave the way to technologically exploit the devices within the aforementioned applications $[11,15]$. The fundamental physics in the discussed devices relies on the efficient spatial and spectral coupling of a single QD to the optical mode of the resonator. Different resonator geometries were traditionally used to study the coupling of single self-assembled QDs to optical resonances, among of which microdisks [16, 17], photonic crystal $(\mathrm{PhC})$ cavities $[8,18-20]$ and micropillar cavities $[21,22]$ are the most prominent. Due to the possibility to reach high quality factors ( $Q$ factors) on the order of $10^{5}[23]$, a highly

\footnotetext{
* corresponding author; e-mail:

sven.hoefling@physik.uni-wuerzburg.de
}

directed vertical emission and the feasibility for current injection in low-mode volume micropillar cavities [15], those structures can be considered as a model system to study important effects in the CQED. Consequently, also the first observation of the Purcell effect between QDs and a monolithic semiconductor cavity mode was reported in a micropillar system [24, 25], reflected by a change of the emission rate of the quantum dot when tuned on resonance with the pillar mode. In fact, the spontaneous emission process is influenced by the presence of the cavity due to the discrete mode spectrum and on resonance the spontaneous emission of the emitter is enhanced by the Purcell factor $F_{\mathrm{P}}$ [1]:

$$
F_{\mathrm{P}}=\frac{3 Q \lambda_{\mathrm{c}}^{3}}{4 \pi^{2} n_{\text {eff }}^{3} V_{\text {mode }}} \frac{\left(\lambda_{\mathrm{c}} / 2\right)^{2}}{\Delta^{2}+\left(\gamma_{\mathrm{c}} / 2\right)^{2}} \frac{|\boldsymbol{E}(\boldsymbol{r})|^{2}}{\left|\boldsymbol{E}_{\max }\right|^{2}} .
$$

This factor strongly depends on cavity parameters such as the $Q$ factor and the mode volume $V_{\text {mode }}$, but also on the spectral QD-cavity detuning $\Delta$ and the spatial position of the quantum dot with respect to the electrical field inside of the micropillar (expressed by $|\boldsymbol{E}(\boldsymbol{r})|^{2}$ ) must be taken into account. While the QD-cavity detuning can be manipulated by means of temperature tuning or more sophisticated techniques as will be presented below, the lateral position of the interacting QD in the devices is left to randomness in most approaches. Possible routes to control the QD position in single micropillar structures include high resolution micro photoluminescence (micro-PL) scanning of a planar sample and subsequent device processing with respect to the QDs $[26,27]$. Yet the fully scalable integration of single QDs in the centre of micropillar resonators is expected to very likely require 
site controlled QD (SCQD) growth and subsequent device alignment $[28,29]$.

By increasing the quality factor $Q$ of the resonators and therefore the photon lifetime $\tau_{\mathrm{p}}\left(\tau_{\mathrm{p}}=\hbar Q / E_{\mathrm{p}}\right)$ within the structures, photon losses can be reduced and the QD-cavity system can eventually be driven into the regime of strong emitter-photon coupling [6, 17, 18, 22, 30, 31]. In this case, the coupled system experiences damped Rabi oscillations reflected in a in the characteristic vacuum Rabi splitting of the emission lines of the coupled QDcavity system under resonant conditions. The two energies of the eigenmodes can then be expressed by the following formula [22]:

$$
E_{1,2}=E_{0}-\mathrm{i}\left(\gamma_{\mathrm{C}}-\gamma_{x}\right) / 4 \pm\left[g^{2}-\left(\gamma_{\mathrm{C}}-\gamma_{x}\right)^{2} / 16\right]^{1 / 2} .
$$

$E_{0}$ denotes the energy of the uncoupled exciton and the cavity mode, $\gamma_{C}$ and $\gamma_{x}$ are the full width at half maximum emission linewidths of the quantum dot and the cavity, respectively. A simple estimation of the threshold of the system for the occurrence of strong coupling in the system can be obtained by setting the square root in the equation equal to zero and neglecting dephasing of the emitter $\left(\gamma_{x} \ll \gamma_{\mathrm{C}}\right)$.

Therefore, strong coupling can be observed in the system if

$$
g \simeq \gamma_{\mathrm{C}} / 4
$$

In this threshold condition and in the equation already given above, $g$ represents the coupling strength of the emitter to the cavity mode, which can be maximized by enhancing the dipole moment of the emitter and reducing the mode volume of the resonator [22].

A benefit of the micropillar cavity geometry is the straightforward way of electrically contacting the devices after sample planarization. In doped structures, electrical current injection could be realized [15] and even ultra-low threshold electrical current lasing effects in micropillars under electrical current injection could be observed [11]. Furthermore, the exploitation of the quantum confined stark Stark effect when applying a vertical electric field to the structures leads to an alternative tuning mechanism of the QD energy [32, 33]. In contrast to temperature tuning, the energy of the excitonic emission can be shifted on a much shorter timescale by an external electrical field with a tuning range comparable to the one achieved via temperature tuning. The potential of this technique is demonstrated in this letter by presenting a strongly coupled QD-resonator system with the emitter being tuned through resonance by the quantum confined Stark effect.

Recently, QD tuning in an external magnetic field applied in the Faraday configuration has attracted interest as the technique allows one not only to blue-shift the excitonic emission energy by the diamagnetic shift but also to directly influence and manipulate the QD oscillator strength [34]. Especially QDs with large surface extensions and therefore rather extended excitonic wave functions exhibit an enhanced shift in the magnetic field up to values of $26 \mu \mathrm{eV} / \mathrm{T}^{2}$, as the altered excitonic eigenfunction energy shift in a magnetic field can be described by [34]:

$$
\Delta E \propto\left\langle\Psi\left|x^{2}+y^{2}\right| \Psi\right\rangle .
$$

Therefore, large QDs with optimized in-plane extensions $x$ and $y$ are good candidates for QD tuning experiments in the magnetic field. Yet, by increasing the magnetic field strength, the extension of the wave function is quenched, and as a consequence, the dipole moment of the QD exciton decreases [34].

The following sections review the advanced techniques of QD manufacturing established in recent years to meet the prerequisites for the discussed experiments. Furthermore, device integration of the QD structures in micropillar cavities is described and selected spectroscopic experiments are presented.

\section{Experimental details and discussion}

\subsection{Quantum dot growth}

The following section briefly summarizes the growth of QDs that were tailored to meet the requirements in the envisaged CQED experiments. For the experiments performed in the strong coupling regime we systematically increased the oscillator strength of the QDs to maximize the light matter coupling strength $g$ for a given mode volume of the resonator. Reducing the indium content in the GaInAs material system to a value of $\approx 30 \%$ in combination with a material deposition just above the critical thickness leads to enlarged surface extension of the quantum dots nanostructures [35]. Furthermore, enhancing the migration length during QD deposition by a rather high substrate temperature of $510^{\circ} \mathrm{C}$ results in dilute QD arrays with densities on the order of $10^{-9} \mathrm{~cm}^{-2}$ as exemplarily depicted in the scanning electron microscope (SEM) image in Fig. 1a of a sample containing uncapped QDs. The dilute area density of the QDs enables the study of single QD effects in the micropillar cavities. The increased volume of the single QDs with elongations of $\approx 100 \mathrm{~nm}$ in the $[0,-1,1]$ direction and $\approx 30 \mathrm{~nm}$ in the $[0,1,1]$ direction significantly increases the QD oscillator strength up to 50, compared to 10-20 for standard single InAs QDs [22, 36].

Additionally, envisaging experiments in a magnetic field in the Faraday configuration, QDs with large surface extensions feature enhanced diamagnetic shifts in the $26 \mu \mathrm{eV} / \mathrm{T}^{2}$ range and therefore pave the way to new single QD tuning techniques.

When a magnetic field is applied parallel to the growth direction, i.e. in Faraday configuration, an additional lateral confinement is provided to the QDs in the scale of the magnetic length. The significant blue shift of the emission wavelength with increasing magnetic field strength (see Fig. 1) is exploited in the experiments described below.

The control of the QD position during growth is a challenging topic and has been accomplished in our group by 

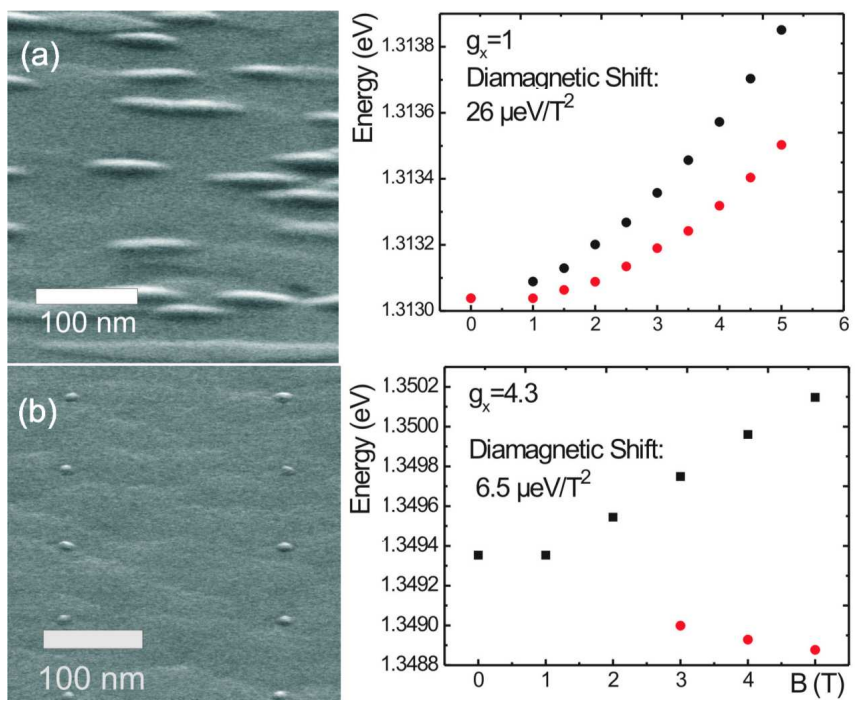

Fig. 1. Scanning electron micrograph of uncapped quantum dots and corresponding photoluminescence shift in a magnetic field: (a) large oscillator strength $\mathrm{GaIn}_{0.3} \mathrm{As}_{0.7} \mathrm{QDs}$; (b) site controlled InAs QDs.

means of directed QD nucleation as demonstrated by various groups [37]. At first nucleation centres are defined on a planar GaAs surface by means of e-beam lithography and etching. After thoroughly removing resists and oxides [38] regrowth on the patterned surface is performed, initialized by a thin GaAs buffer layer to smooth the surface and subsequent deposition of InAs QDs in single layers or stacked QD layers at low growth rates. This technique allowed us to direct the nucleation of the InAs QDs to a high degree as shown in scanning electron microscopy images on uncapped QDs in Fig. 1. Details on the growth of the site controlled quantum dots can be found in $[28,29]$ and will be briefly reviewed. The single site controlled InAs QDs described in this letter were grown on stacks of two InAs layers to increase the distance of the QD to the regrowth surface and to reduce the detrimental effect of the etched pits in the regrowth surface on the optical properties of the single QDs. The combination of site controlled QD growth with a careful device alignment enabled us to deterministically implement single QDs in the centres of micropillar cavities in a fully scalable manner. We also probed the QDs in a magnetic field to assess the diamagnetic shift of the SCQDs. In contrast to the $\mathrm{Ga}_{0.7} \mathrm{In}_{0.3} \mathrm{As}$ QDs, the diamagnetic shift of the SCQDs ranges around $6 \mu \mathrm{eV} / \mathrm{T}^{2}$ which is similar to previous studies on single InAs QDs. We also observed a rather large Zeeman splitting of the SCQDs with a $g_{x}$-factor value of up to 4 also comparing well with previous investigation on standard StranskiKrastanov InAs QDs [39].

\subsection{Quantum dot integration in micropillar cavities}

For the device integration of QDs into micropillar cavities a single dilute layer of ( $\mathrm{Ga}) \mathrm{InAs}$ QDs is positioned in the vertical field maximum of a one lambda cavity. The GaAs cavity layer is sandwiched between two highly reflecting AlAs/GaAs distributed Bragg reflector (DBR) mirrors with up to 32 mirror pairs in the top and 36 mirror pairs in the bottom DBR. The planar resonance of the structures is tailored to match the QD photoluminescence in a manner such that QDs on the low energy tail of the ensemble spectrum featuring the largest oscillator strength and moderate spectral QD densities lower than one QD emission line per $\mathrm{meV}$ were envisaged for the resonance tuning experiments. Subsequently, micropillars are defined by e-beam lithography and etched into the sample by a reactive ion etch treatment [23]. As optimization of the sample growth and processing such as described in [23] leads to cavity $Q$ factors exceeding 150000 for undoped structures and 16000 [15] for doped pillars.

To electrically address the QDs in the structures, sample planarization and contacting of the pillars as described in [15] are performed. Ring shaped gold contacts are aligned with respect to the micropillars allowing for an efficient light outcoupling of the devices while allowing to homogeneously electrically pump or manipulate the device by electric fields.

For the device integration of the SCQDs in micropillar cavities, a stack of positioned InAs quantum dots with three spectrally detuned InAs layers was integrated into the resonators. Subsequently the micropillars were defined relatively to the SCQD positions ensuring each device features not more than one detuned QD stack in its centre.

\subsection{CQED experiments in the strong coupling regime}

In the following section, experiments in the strong coupling regime under optical excitation are discussed. All devices feature a single layer of GaInAs QDs integrated in high $Q$-factor micropillar cavities. The QDs were pumped by a frequency-doubled Nd:YAG laser at $532 \mathrm{~nm}$ above the GaAs band edge in the cavity and the signal is collected by a double monochromator or a high resolution monochromator to allow for spectral resolution better than $20 \mu \mathrm{eV}$.

In Fig. 2a temperature tuning of the QD emission line was employed to achieve resonance of the QD and the cavity mode with a $Q$ factor of 11000 (pillar diameter $1.6 \mu \mathrm{m})$.

Owing to the enhanced oscillator strength of the $\mathrm{Ga}_{0.3} \mathrm{In}_{0.7} \mathrm{As}$ QDs and the high quality factor of the micropillar combined with an optical mode volume of $\approx 0.3 \mu \mathrm{m}^{3}$ the required threshold to reach the strong coupling regime could be overcome. The system exhibits two distinct peaks at resonance which can be attributed to the eigenenergies of a coupled QD-microcavity system in the strong coupling regime. In fact, the characteristic anticrossing of the excitonic emission line and the cavity mode with a vacuum Rabi splitting of $105 \mu \mathrm{eV}$ was observed in the discussed system.

Tuning the excitonic emission line by means of temperature is a feasible tool to probe the principle physical 


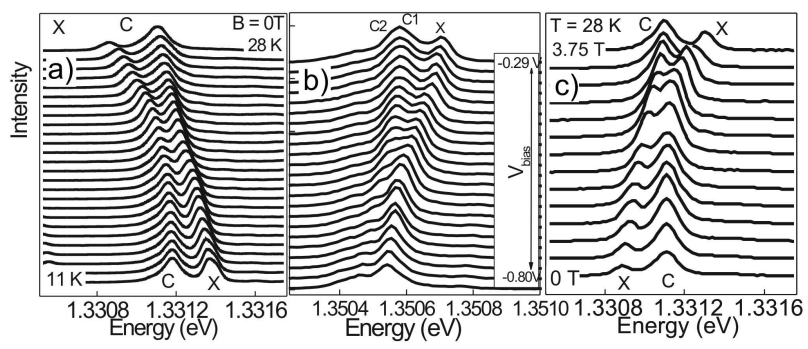

Fig. 2. Different tuning techniques applied to sweep a single quantum dot emission line through a high $Q$ micropillar cavity mode in the regime of strong coupling: (a) temperature tuning, (b) electro-optical tuning, (c) magneto-optical tuning.

effects, as it does not require further steps of sample processing. Yet, the tuning range of the GaInAs QDs is rather limited to values in the $1-2 \mathrm{meV}$ range before the emission is quenched due to enhanced thermionic emission of confined carriers and furthermore the tuning of the QD emission line takes typically several seconds before the sample reaches its thermal equilibrium.

To reduce the timescale of the tuning mechanism (SPEC), the quantum confined Stark effect can be exploited for instance to sweep the QD emission. This requires electrical contacting of the micropillar sample in the previously mentioned manner. The sample was pumped optically with a continuous wave laser operating at $890 \mathrm{~nm}$, e.g. below the GaAs bandgap, in order to avoid absorption in the doped DBR. The emission of the sample was collected by the objective, while the sample is electrically addressed. Figure $2 \mathrm{~b}$ shows a compilation of spectra taken under increasing reverse bias of the devices. The $\mathrm{QD}$ emission is tuned to longer wavelength by increasing the voltage, while the cavity resonance remains at the same spectral position. Again, a pronounced anticrossing of the associated with a vacuum Rabi splitting of $\mathrm{QD}$ and the fundamental cavity mode $(Q: 14000)$ amounting to $63 \mu \mathrm{eV}$ is observed, showing the maturity of the device processing and the feasibility of reaching the strong coupling regime in electrically addressed QDmicropillar cavities.

As a further tuning mechanism of the QD emission one can also employ the shift of QD excitons in a magnetic field. As discussed in [34, 39], this shift is fairly pronounced in standard InAs quantum dots in a manner comparable to Fig. 1b. In our high oscillator strength $\mathrm{In}_{0.3} \mathrm{Ga}_{0.7} \mathrm{As}$ QDs, the large lateral extension of the excitonic wave function results in a particular high diamagnetic coefficient which facilitates magneto-optical tuning in the strong coupling with a tunability of the QD emission line in the meV range comparable to temperature or Stark shift tuning techniques.

Magneto-optical resonance tuning was exploited in Fig. 2c, where a QD is swept through the fundamental cavity mode by its diamagnetic shift. A vacuum Rabi splitting amounting to $95 \mu \mathrm{eV}$ could be extracted.
Interestingly, this allows for an in situ manipulation of the oscillator strength which decreases for large fields as the additional degree of confinement provided the magnetic field quenches the extension of the excitonic wave function [34].

\subsection{Deterministic light matter interaction with single positioned QDs}

To exploit a coupled QD to a cavity resonance as an efficient source of single photons, one has to ensure that only a limited amount of QDs emit in the spectral vicinity of the cavity resonance to prevent several QDs from contributing to the cavity emission. Even the uncorrelated background light of rather far detuned QDs can be funneled in the cavity mode $[5,6]$ and prevents the observation of single photon emission with $g^{(2)}(0)$ values in high quality factor cavities down to 0 .

Therefore, the integration of no more than a single QD in the device is desirable, and furthermore an efficient QD-cavity coupling can only occur if the dot is located in the centre of the resonator device.

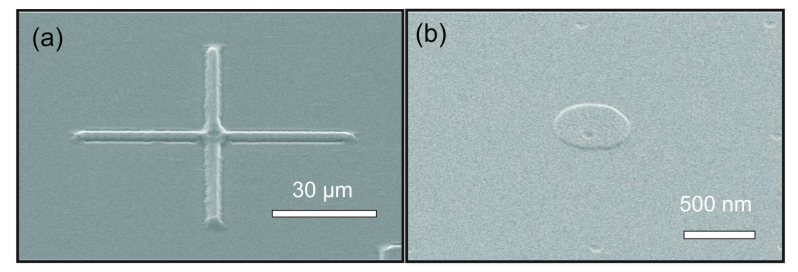

Fig. 3. (a) Alignment marks for quantum dot and device alignment overgrown with $2 \mu \mathrm{m}$ of GaAs. (b) Alignment of two subsequent e-beam steps after overgrowth.

We have employed site controlled QD growth to ensure a rather accurate position control of a single QD. The position control of our QDs was estimated to values below $50 \mathrm{~nm}$ ensuring a good addressability of the SCQDs [28]. Furthermore, device alignment of the micropillar devices was investigated on overgrown samples. The micropillar devices and the QD nucleation centres were aligned with respect to the same alignment marks on the sample in the subsequent processing steps. As the thickness of the top DBR section with 12 mirror pairs amounts to $2 \mu \mathrm{m}$, the alignment marks slightly smear out after overgrowth (Fig. 3a). This makes the integration process much more challenging for micropillar cavities compared to photonic crystal cavities which has consequently been demonstrated beforehand [40]. Yet, the smeared out alignment marks were still detectable in the e-beam system, but the alignment accuracy was decreased to a value of $\pm 100 \mathrm{~nm}$ (Fig. 3b). Taking this accuracy into account we aligned micropillar cavities with diameters of down to $1 \mu \mathrm{m}$ with respect to the QDs. Each micropillar contains a single stack of three spectrally detuned InAs layers, one of which finds it shows PL emission line in the spectral range around 950-970 $\mathrm{nm}$ and was envisaged for further experiments. 
Figure 4a shows spectra recorded under varying excitations. The spectrally detuned QD1 is shifted to an emission wavelength of $929 \mathrm{~nm}$ by partial capping and in situ annealing during growth. QD2 was not annealed within the QD stack, hence the emission is shifted to the red side. In the presented device, the emission lines attributed to QD2 could be brought in resonance with the cavity mode and therefore their intensity is brighter due to the Purcell effect.

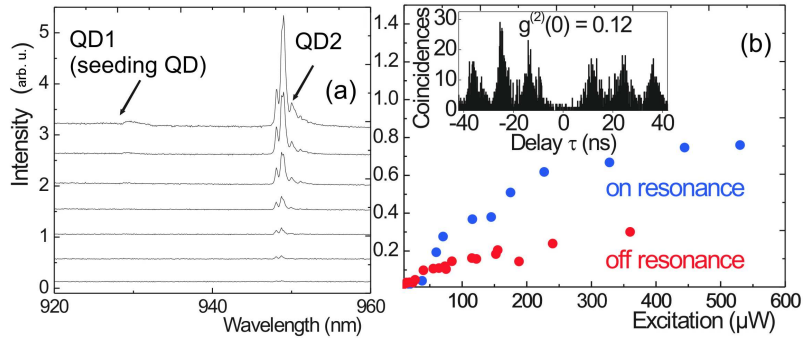

Fig. 4. (a) Excitation series of a site controlled quantum dot stack (QD2 and QD1 in subsequent layers) on resonance with a micropillar cavity mode. (b) Comparison of the emission intensities with the SCQD tuned onand off resonance. Inset: pulsed $g^{(2)}(0)$ measurement with the SCQD on resonance with the cavity mode.

The integrated intensities of this QD when tuned on and off resonance are compared in Fig. 4b. Obviously, when the SCQD is off resonance at $10 \mathrm{~K}$, the emission saturates at a level which is reduced by a factor of 2.5 when compared to the on resonance case. This indicates the accomplished spectral coupling of the SCQD with the cavity mode.

On resonance, photon correlation measurements were performed at a Hanbury-Brown and Twiss setup to probe the feasibility to operate the system as a triggered source of single photons. The QD was excited above the GaAs band edge by a pulsed titan-sapphire laser.

The photon statistics of the system is shown in the inset of Fig. 4b, clearly pointing out the reduced peak at zero time delay. The $g^{(2)}(0)$ value was extracted to be 0.12 , proving the single photon emission behavior of the coupled SCQD-resonator system.

In order to reach the regime of strong coupling with those kind of positioned quantum dots, the optical purity of the QD emitters must be improved as typically the single QD linewidth of site controlled QDs grown on GaAs etched nanoholes exceeds the herewith presented values for the vacuum Rabi splitting energies. Record linewidth for SCQDs down to values of $18 \mu \mathrm{eV}$ have, however, very recently been published [41] which is an encouraging result towards the scalable and deterministic realization of strongly coupled SCQD resonators.

\section{Conclusions}

CQED experiments with single QDs were performed in micropillar resonators. The regime of strong light- -emitter coupling was reached by implementing high oscillator strength GaInAs quantum dots in high finesse micropillar resonators. The QD emission lines were tuned via several techniques through resonance, namely by temperature, the quantum confined Stark effect and the spectral shift in an external magnetic field. With all employed techniques, the characteristic anticrossing of exciton and cavity mode could be observed.

Furthermore, deterministic spatial resonance of single QDs in micropillars could be reached by combining site controlled quantum dot growth and device alignment. The weak coupling regime of a single SCQD and a moderate $Q$ cavity mode could be exploited to enhance the intensity of the SCQD emission line and lead to single photon emission.

\section{Acknowledgments}

The authors thank M. Emmerling and T. Steinl for their assistance in sample preparation. This work has been financially supported by the German Ministry of Education and Research within the project "QPENS" and the European Commission within the projects "QPhoton".

\section{References}

[1] J.M. Gerard, in: Single Quantum Dots: Physics and Applications, Ed. P. Michler, Topics of Applied Physics, Vol. 30, Springer, Heidelberg 2003, p. 269.

[2] A.J. Shields, Nature Photon. 1, 215 (2007).

[3] M. Pelton, C. Santori, J. Vuckovic, B.Y. Zhang, G.S. Solomon, J. Plant, Y. Yamamoto, Phys. Rev. Lett. 89, 233602 (2002).

[4] E. Moreau, I. Robert, L. Manin, V. Thierry-Mieg, J.M. Gerard, I. Abram, Physica E Low-Dimens. Syst. Nanostruct. 13, 418 (2002).

[5] D. Press, S. Götzinger, S. Reitzenstein, C. Hofmann, A. Loffler, M. Kamp, A. Forchel, Y. Yamamoto, Phys. Rev. Lett. 98, 117402 (2007).

[6] K. Hennessy, A. Badolato, M. Winger, D. Gerace, M. Atatüre, S. Gulde, S. Fält, E.L. Hu, A. Imamoglu, Nature Photon. 1, 215 (2007).

[7] S. Strauf, N.G. Stoltz, M.T. Rakher, L.A. Coldren, P.M. Petroff, D. Bouwmeester, Nature Photon. 1, 704 (2007).

[8] S. Strauf, K. Hennessy, M.T. Rakher, Y.-S. Choi, A. Badolato, L.C. Andreani, E.L. Hu, P.M. Petroff, D. Bouwmeester, Phys. Rev. Lett. 96, 127404 (2006).

[9] Z.G. Xie, S. Götzinger, W. Fang, H. Cao, G.S. Solomon, Phys. Rev. Lett. 98, 117401 (2007).

[10] S. Reitzenstein, C. Böckler, A. Bazhenov, A. Gorbunov, A. Löffler, M. Kamp, V.D. Kulakovskii, A. Forchel, Opt. Express 16, 4848 (2008).

[11] S. Reitzenstein, T. Heindel, C. Kistner, A. Rahimi-Iman, C. Schneider, S. Hofling, A. Forchel, Appl. Phys. Lett. 93, 061104 (2008)

[12] D. Press, T.D. Ladd, B.Y. Zhang, Y. Yamamoto, $N a-$ ture 456, 218 (2008). 
[13] A. Imamoglu, D.D. Awschalom, G. Burkard, D.P. DiVincenzo, D. Loss, M. Sherwin, A. Small, Phys. Rev. Lett. 83, 4204 (1999).

[14] C.Y. Hu, W.J. Munro, J.G. Rarity, Phys. Revi. B 83, 78, 125318 (2008).

[15] C. Böckler, S. Reitzenstein, C. Kistner, R. Debusmann, A. Löffler, T. Kida, S. Höfling, A. Forchel, L. Grenouillet, J. Claudon, J.M. Gérard, Appl. Phys. Lett. 92, 091107 (2008).

[16] P. Michler, A. Kiraz, C. Becher, W.V. Schoenfeld, P.M. Petroff, L.D. Zhang, E. Hu, A. Imamoglu, Science 290, 2282 (2000).

[17] E. Peter, P. Senellart, D. Martrou, A. Lemaitre, J. Hours, J.M. Gerard, J. Bloch, Phys. Rev. Lett. 95, 067401 (2005).

[18] T. Yoshie, A. Scherer, J. Hendrickson, G. Khitrova, H.M. Gibbs, G. Rupper, C. Ell, O.B. Shchekin, D.G. Deppe, Nature 432, 200 (2004).

[19] D. Englund, A. Faraon, I. Fushman, N. Stoltz, P. Petroff, J. Vuckovic, Nature 450, 857 (2007).

[20] M. Nomura, Y. Ota, N. Kumagai, S. Iwamoto, Y. Arakawa, Appl. Phys. Express 1, 072102 (2008).

[21] J.M. Gerard, D. Barrier, J.Y. Marzin, R. Kuszelewicz, L. Manin, E. Costard, V. Thierry-Mieg, T. Rivera, Appl. Phys. Lett. 69, 449 (1996).

[22] J.P. Reithmaier, G. Sek, A. Loffler, C. Hofmann, S. Kuhn, S. Reitzenstein, L.V. Keldysh, V.D. Kulakovskii, T.L. Reinecke, A. Forchel, Nature 432, 197 (2004).

[23] S. Reitzenstein, C. Hofmann, A. Gorbunov, M. Gorbunov, M. Strauß, S.H. Kwon, C. Schneider, A. Loffler, S. Hofling, M. Kamp, A. Forchel, Appl. Phys. Lett. 90, 251109 (2007).

[24] J.M. Gerard, B. Sermage, B. Gayral, B. Legrand, E. Costard, V. Thierry-Mieg, Phys. Rev. Lett. $\mathbf{8 1}$ 1110 (1998).

[25] G.S. Solomon, M. Pelton, Y. Yamamoto, Phys. Status Solidi A-Appl. Res. 178, 341 (2000).

[26] A. Dousse, L. Lanco, J. Suffczynski, E. Semenova, A. Miard, A. Lemaitre, I. Sagnes, C. Roblin, J. Bloch, P. Senellart, Phys. Rev. Lett. 101, 267404 (2008).

[27] A. Dousse, J. Suffczyński, R. Braive, A. Miard, A. Lemaître, I. Sagnes, L. Lanco, J. Bloch, P. Voisin, P. Senellart, Appl. Phys. Lett. 94, 121102 (2009).
[28] C. Schneider, M. Strauss, T. Sünner, A. Huggenberger, D. Wiener, S. Reitzenstein, M. Kamp, S. Höfling, A. Forchel, Appl. Phys. Lett. 92, 183101 (2008).

[29] C. Schneider, T. Heindel, A. Huggenberger, P. Weinmann, C. Kistner, M. Kamp, S. Reitzenstein, S. Höfling, A. Forchel, Appl. Phys. Lett. 94, 111111 (2009).

[30] L.C. Andreani, G. Panzarini, J.M. Gerard, Phys. Rev. B 60, 13276 (1999).

[31] G. Khitrova, H.M. Gibbs, M. Kira, S.W. Koch, A. Scherer, Nature Phys. 2, 81 (2006).

[32] C. Kistner, T. Heindel, C. Schneider, A. Rahimi-Iman, S. Reitzenstein, S. Hofling, A. Forchel, Opt. Express 16, 15006 (2008).

[33] A. Laucht, F. Hofbauer, N. Hauke, J. Angele, S. Stobbe, M. Kaniber, G. Bohm, P. Lodahl, M.C. Amann, J.J. Finley, New J. Phys. 11, 023034 (2009).

[34] S. Reitzenstein, S. Munch, P. Franeck, A. Rahimi-Iman, A. Löffler, S. Höfling, L. Worschech, A. Forchel, Phys. Rev. Lett. 103, 127401 (2009).

[35] A. Löffler, J.P. Reithmaier, G. Seak, C. Hofmann, S. Reitzenstein, M. Kamp, A. Forchel, Appl. Phys. Lett. 86, 111105 (2005).

[36] J. Johansen, S. Stobbe, I.S. Nikolaev, T. Lund-Hansen, P.T. Kristensen, J.M. Hvam, W.L. Vos, P. Lodahl, Phys. Rev. B 77, 073303 (2008).

[37] O.G. Schmidt, Lateral Alignment of Epitaxial Quantum Dots, Springer, Berlin 2007.

[38] C. Schneider, A. Huggenberger, T. Sünner, T. Heindel, M. Strauß, S. Göpfert, P. Weinmann, S. Reitzenstein, L. Worschech, M. Kamp, S. Höfling, A. Forchel, Nanotechnology, 20, 434012 (2009).

[39] M.F. Tsai, H. Lin, C.H. Lin, S.D. Lin, S.Y. Wang, M.C. Lo, S.J. Cheng, M.C. Lee, W.H. Chang, Phys. Rev. Lett. 101, 267402 (2008).

[40] T. Sünner, C. Schneider, M. Strauß, A. Huggenberger, D. Wiener, S. Höfling, M. Kamp, A. Forchel, Opt Lett. 33, 1759 (2008).

[41] L.O. Mereni, V. Dimastrodonato, R.J. Young, E. Pelucchi, Appl. Phys. Lett. 94, 223121 (2009). 